ANNALES

POLONICI MATHEMATICI

$82.2(2003)$

\title{
Extension theorems of Sakai type for separately holomorphic and meromorphic functions
}

\author{
by Peter Pflug and Viêt Anh Nguyên (Oldenburg)
}

\begin{abstract}
We first exhibit counterexamples to some open questions related to a theorem of Sakai. Then we establish an extension theorem of Sakai type for separately holomorphic/meromorphic functions.
\end{abstract}

1. Introduction. We first fix some notations and terminology. Throughout the paper, $E$ denotes the unit disc of $\mathbb{C}$, and for any set $S \subset \mathbb{C}^{n}$, int $S$ (or equivalently $\operatorname{int}_{\mathbb{C}^{n}} S$ ) denotes the interior of $S$. For any domain $D \subset \mathbb{C}^{n}$, we say that the subset $S \subset D$ does not separate domains in $D$ if for every domain $U \subset D$, the set $U \backslash S$ is connected. Moreover, $\mathcal{O}(D)($ resp. $\mathcal{M}(D)$ ) will denote the space of holomorphic (resp. meromorphic) functions on $D$. Finally, if $S$ is a subset of $D \times G$, where $D \subset \mathbb{C}^{p}, G \subset \mathbb{C}^{q}$ are some open sets, then for $a \in D$ (resp. $b \in G$ ), the fiber $S(a, \cdot)$ (resp. $S(\cdot, b)$ ) is the set $\{w \in G:(a, w) \in S\}$ (resp. $\{z \in D:(z, b) \in S\}$ ).

In 1957 E. Sakai [9] claimed to have proved the following result.

TheOREM. Let $S \subset E \times E$ be a relatively closed set such that int $S=\emptyset$ and $S$ does not separate domains in $E \times E$. Let $A$ (resp. B) be the set of all $a \in E($ resp. $b \in E)$ such that $\operatorname{int}_{\mathbb{C}} S(a, \cdot)=\emptyset\left(\right.$ resp. $\left.\operatorname{int}_{\mathbb{C}} S(\cdot, b)=\emptyset\right)$. Put $X:=\mathbb{X}(A, B ; E, E)=(A \times E) \cup(E \times B)$. Then for every function $f: X \backslash S \rightarrow \mathbb{C}$ which is separately meromorphic on $X$, there exists an $\widehat{f} \in \mathcal{M}(E \times E)$ such that $\widehat{f}=f$ on $X \backslash S$.

Unfortunately, it turns out, as reported in [4], that the proof of E. Sakai contains an essential gap. In the latter paper M. Jarnicki and the first author gave a correct proof of this theorem.

E. Sakai also claimed in [9] that the following question (the $n$-dimensional version of the Theorem) can be answered positively but he did not give any proof.

2000 Mathematics Subject Classification: Primary 32D15, 32D10.

Key words and phrases: Cross Theorem, holomorphic/meromorphic extension, envelope of holomorphy. 
QUESTION 1. For any $n \geq 3$, let $S \subset E^{n}$ be a relatively closed set such that int $S=\emptyset$ and $S$ does not separate domains. Let $f: E^{n} \backslash S \rightarrow \mathbb{C}$ be such that for any $j \in\{1, \ldots, n\}$ and for any $\left(a^{\prime}, a^{\prime \prime}\right) \in E^{j-1} \times E^{n-j}$ with $\operatorname{int}_{\mathbb{C}} S\left(a^{\prime}, \cdot, a^{\prime \prime}\right)=\emptyset$, the function $f\left(a^{\prime}, \cdot, a^{\prime \prime}\right)$ extends meromorphically to $E$. Does $f$ always extend meromorphically to $E^{n}$ ?

In connection with the Theorem and Question 1, M. Jarnicki and the first author [4] posed two more questions:

QUESTION 2. Let $\mathcal{A}$ be a subset of $E^{n}(n \geq 2)$ which is plurithin at $0 \in E^{n}$ (see Section 2 below for the definition). For an arbitrary open neighborhood $U$ of 0 , does there exist a non-empty relatively open subset $C$ of a real hypersurface in $U$ such that $C \subset U \backslash \mathcal{A}$ ?

Question 3. Let $D \subset \mathbb{C}^{p}, G \subset \mathbb{C}^{q}(p, q \geq 2)$ be pseudoconvex domains and let $S \subset D \times G$ be a relatively closed set such that int $S=\emptyset$ and $S$ does not separate domains in $D \times G$. Let $A$ (resp. B) be the set of all $a \in D$ $($ resp. $b \in G)$ such that $\operatorname{int}_{\mathbb{C}^{q}} S(a, \cdot)=\emptyset\left(\right.$ resp. $\left.\operatorname{int}_{\mathbb{C}^{p}} S(\cdot, b)=\emptyset\right)$. Put $X:=$ $\mathbb{X}(A, B ; D, G)=(A \times G) \cup(D \times B)$ and let $f: X \backslash S \rightarrow \mathbb{C}$ be separately meromorphic on $X$. Does there always exist a function $\widehat{f} \in \mathcal{M}(D \times G)$ such that $\widehat{f}=f$ on $X \backslash S$ ?

This note has two purposes. The first one is to give counterexamples to the three open questions above. The second one is to describe the maximal domain to which the function $f$ in Questions 1 and 3 can be meromorphically extended.

The paper is organized as follows. We begin Section 2 by collecting some background of pluripotential theory and introducing some notations. This is necessary to state the results afterwards.

Section 3 provides three counterexamples to the three open questions above.

The subsequent sections are devoted to the proof of a result in the positive direction. More precisely, we describe qualitatively the maximal domain of meromorphic extension of the function $f$ in Questions 1 and 3. Section 4 develops auxiliary tools that will be used in Section 5 to prove the positive result.

Acknowledgments. The paper was written while the second author was visiting the Carl von Ossietzky Universität Oldenburg, being supported by the Alexander von Humboldt Foundation. He wishes to express his gratitude to these organisations.

2. Background and statement of the results. We keep the main notation from [4]. 
Let $N \in \mathbb{N}, N \geq 2$, and let $\emptyset \neq A_{j} \subset D_{j} \subset \mathbb{C}^{n_{j}}$, where $D_{j}$ is a domain, $j=1, \ldots, N$. We define an $N$-fold cross

$$
\begin{aligned}
X & =\mathbb{X}\left(A_{1}, \ldots, A_{N} ; D_{1}, \ldots, D_{N}\right) \\
& :=\bigcup_{j=1}^{N} A_{1} \times \ldots \times A_{j-1} \times D_{j} \times A_{j+1} \times \ldots \times A_{N} \subset \mathbb{C}^{n_{1}+\ldots+n_{N}}=\mathbb{C}^{n} .
\end{aligned}
$$

For an open set $\Omega \subset \mathbb{C}^{n}$ and $A \subset \Omega$, put

$$
h_{A, \Omega}:=\sup \{u: u \in \operatorname{PSH}(\Omega), u \leq 1 \text { on } \Omega, u \leq 0 \text { on } A\},
$$

where $\operatorname{PSH}(\Omega)$ denotes the set of all plurisubharmonic functions on $\Omega$. Put

$$
\omega_{A, \Omega}:=\lim _{k \rightarrow \infty} h_{A \cap \Omega_{k}, \Omega_{k}}^{*}
$$

where $\left\{\Omega_{k}\right\}_{k=1}^{\infty}$ is a sequence of relatively compact open sets $\Omega_{k} \subset \Omega_{k+1} \subset \Omega$ with $\bigcup_{k=1}^{\infty} \Omega_{k}=\Omega\left(h^{*}\right.$ denotes the upper semicontinuous regularization of $\left.h\right)$. We say that a subset $\emptyset \neq A \subset \mathbb{C}^{n}$ is locally pluriregular if $h_{A \cap \Omega, \Omega}^{*}(a)=0$ for any $a \in A$ and for any open neighborhood $\Omega$ of $a$. We say that $A$ is plurithin at a point $a \in \mathbb{C}^{n}$ if either $a \notin \bar{A}$, or $a \in \bar{A}$ and $\lim _{\sup _{A \backslash\{a\} \ni z \rightarrow a} u(z)<u(a)}$ for a suitable function $u$ plurisubharmonic in a neighborhood of $a$. For a good background of pluripotential theory, see the books [5] or [1].

For an $N$-fold cross $X:=\mathbb{X}\left(A_{1}, \ldots, A_{N} ; D_{1}, \ldots, D_{N}\right)$ let

$$
\widehat{X}:=\left\{\left(z_{1}, \ldots, z_{N}\right) \in D_{1} \times \ldots \times D_{N}: \sum_{j=1}^{N} \omega_{A_{j}, D_{j}}\left(z_{j}\right)<1\right\} .
$$

Suppose that $S_{j} \subset\left(A_{1} \times \ldots \times A_{j-1}\right) \times\left(A_{j+1} \times \ldots \times A_{N}\right), j=1, \ldots, N$. Define the generalized $N$-fold cross

$$
\begin{aligned}
T & =\mathbb{T}\left(A_{1}, \ldots, A_{N} ; D_{1}, \ldots, D_{N} ; S_{1}, \ldots, S_{N}\right) \\
& :=\bigcup_{j=1}^{N}\left\{\left(z^{\prime}, z_{j}, z^{\prime \prime}\right) \in\left(A_{1} \times \ldots \times A_{j-1}\right) \times D_{j} \times\left(A_{j+1} \times \ldots \times A_{N}\right):\right. \\
& \left.\left(z^{\prime}, z^{\prime \prime}\right) \notin S_{j}\right\} .
\end{aligned}
$$

Let $M \subset T$ be a relatively closed set. We say that a function $f: T \backslash M$ $\rightarrow \mathbb{C}$ is separately holomorphic and write $f \in \mathcal{O}_{\mathrm{S}}(T)$ (resp. separately meromorphic and write $\left.f \in \mathcal{M}_{\mathrm{S}}(T)\right)$ if for any $j \in\{1, \ldots, N\}$ and $\left(a^{\prime}, a^{\prime \prime}\right) \in$ $\left(A_{1} \times \ldots \times A_{j-1}\right) \times\left(A_{j+1} \times \ldots \times A_{N}\right) \backslash S_{j}$ the function $f\left(a^{\prime}, \cdot, a^{\prime \prime}\right)$ can be holomorphically (resp. meromorphically) extended to $D_{j}\left({ }^{1}\right)$.

We are now ready to state the results. The following propositions give negative answers to Questions 2, 3 and 1 respectively.

$\left({ }^{1}\right)$ Observe that we have changed a little the definition appearing in [2]-[4]. 
Proposition A. For any $n \geq 2$, there is an open dense subset $\mathcal{A}$ of $E^{n}$ which is plurithin at 0 and there exists no non-empty relatively open subset $C$ of a real hypersurface such that $C \subset E^{n} \backslash \mathcal{A}$.

Proposition B. Let $D \subset \mathbb{C}^{p}, G \subset \mathbb{C}^{q}(p, q \geq 2)$ be bounded pseudoconvex domains. Then there is a relatively closed set $S \subset D \times G$ with the following properties:

(i) int $S=\emptyset$ and $S$ does not separate domains;

(ii) let $A$ (resp. $B)$ be the set of all $a \in D$ (resp. $b \in G$ ) such that $\operatorname{int}_{\mathbb{C}^{q}} S(a, \cdot)=\emptyset\left(\right.$ resp. $\left.\operatorname{int}_{\mathbb{C}^{p}} S(\cdot, b)=\emptyset\right)$ and put $X:=\mathbb{X}(A, B ; D, G)$; then there exists a function $f: X \backslash S \rightarrow \mathbb{C}$, separately holomorphic on $X$, such that there is no function $\widehat{f} \in \mathcal{M}(D \times G)$ with $\widehat{f}=f$ on $X \backslash S$.

Proposition C. For all $n \geq 3$, there is a relatively closed set $S \subset E^{n}$ with the following properties:

(i) int $S=\emptyset$ and $S$ does not separate domains;

(ii) for $1 \leq j \leq n$, let $S_{j}$ denote the set of all $\left(a^{\prime}, a^{\prime \prime}\right) \in E^{j-1} \times E^{n-j}$ such that $\operatorname{int}_{\mathbb{C}} S\left(a^{\prime}, \cdot, a^{\prime \prime}\right) \neq \emptyset$ and define the $n$-fold generalized cross $T:=$ $\mathbb{T}\left(E, \ldots, E ; E, \ldots, E ; S_{1}, \ldots, S_{n}\right)$; then there is a function $f: T \backslash S \rightarrow \mathbb{C}$ which is separately holomorphic on $T$ and there is no function $\widehat{f} \in \mathcal{M}\left(E^{n}\right)$ such that $\widehat{f}=f$ on $T \backslash S$.

Problem. Are the answers to Questions 1 and 3 affirmative if the condition on $S$ is sharpened as follows: $S$ does not separate lower-dimensional domains?

Finally, we state a result in the positive direction.

Theorem D. For all $j \in\{1, \ldots, N\} \quad(N \geq 2)$, let $D_{j}$ be a pseudoconvex domain in $\mathbb{C}^{n_{j}}$ and let $S$ be a relatively closed set of $D:=D_{1} \times \ldots \times D_{N}$ with $\operatorname{int} S=\emptyset$. For $j \in\{1, \ldots, N\}$, let $S_{j}$ denote the set of all $\left(a^{\prime}, a^{\prime \prime}\right) \in\left(D_{1} \times\right.$ $\left.\ldots \times D_{j-1}\right) \times\left(D_{j+1} \times \ldots \times D_{N}\right)$ such that $\operatorname{int}_{\mathbb{C}^{n_{j}}} S\left(a^{\prime}, \cdot, a^{\prime \prime}\right) \neq \emptyset$ and define the $N$-fold generalized cross $T:=\mathbb{T}\left(D_{1}, \ldots, D_{N} ; D_{1}, \ldots, D_{N} ; S_{1}, \ldots, S_{N}\right)$. Let $f: T \backslash S \rightarrow \mathbb{C}$ be a function such that $f \in \mathcal{O}_{\mathrm{s}}(T)$ (resp. $f \in \mathcal{M}_{\mathrm{s}}(T)$ ).

(i) Then there are an open dense set $\Omega$ of $D$ and exactly one function $\widehat{f} \in \mathcal{O}(\Omega)$ such that $\widehat{f}=f$ on $(T \cap \Omega) \backslash S$.

(ii) In the case where $N=2$, (i) can be strengthened as follows. Let $\Omega_{j}$ be a relatively compact pseudoconvex subdomain of $D_{j}(j=1,2)$. Then there are an open dense set $A_{j}$ in $\Omega_{j}$ and exactly one function $\widehat{f} \in \mathcal{O}(\widehat{X})$ (resp. $\widehat{f} \in \mathcal{M}(\widehat{X}))$, where $X:=\mathbb{X}\left(A_{1}, A_{2} ; \Omega_{1}, \Omega_{2}\right)$, such that $\widehat{f}=f$ on $(T \cap \widehat{X}) \backslash S$.

A remark is in order. In contrast with the other usual extension theorems (see [1]-[4] and the references therein), the domain of meromorphic/holomorphic extension of the function $f$ in Theorem $\mathrm{D}$ depends on $f$. 
3. Three counterexamples. In what follows we will fix a function $v \in$ $\mathcal{S H}(2 E)$ such that $v(0)=0$ and the complete polar set $\{z \in 2 E: v=-\infty\}$ is dense in $2 E$. For example one can choose $v$ of the form

$$
v(z):=\sum_{k=1}^{\infty} \frac{\log \left(\left|z-q_{k}\right| / 4\right)}{d_{k}}-\sum_{k=1}^{\infty} \frac{\log \left(\left|q_{k}\right| / 4\right)}{d_{k}},
$$

where $(\mathbb{Q}+i \mathbb{Q}) \cap 2 E^{*}=\left\{q_{1}, q_{2}, \ldots, q_{k}, \ldots\right\}, E^{*}:=E \backslash\{0\}$, and $\left\{d_{k}\right\}_{k=1}^{\infty}$ is any sequence of positive real numbers such that $\sum_{k=1}^{\infty} \frac{\log \left(\left|q_{k}\right| / 4\right)}{d_{k}}$ is finite.

For any positive integer $n \geq 2$, define a new function $u \in \operatorname{PSH}\left((2 E)^{n}\right)$ and a subset $\mathcal{A}$ of $E^{n}$ as follows:

$$
\begin{aligned}
& u(z):=\sum_{k=1}^{n} v\left(z_{k}\right), \quad z=\left(z_{1}, \ldots, z_{n}\right) \in(2 E)^{n}, \\
& \mathcal{A}=\mathcal{A}_{n}:=\left\{z \in E^{n}: u(z)<-1\right\} .
\end{aligned}
$$

Observe that $\mathcal{A}$ is an open dense subset of $E^{n}$ because $\mathcal{A}$ contains the set $\{z \in E: v=-\infty\} \times \ldots \times\{z \in E: v=-\infty\}$, which is dense in $E^{n}$ by our construction (3.1) above.

Proposition 3.1. Let $\mathcal{S}$ be any closed set contained in the closed set $E^{n} \backslash \mathcal{A}$. Then $\mathcal{S}$ does not separate domains.

Taking this proposition for granted, we are now able to complete the proof of Proposition A.

Proof of Proposition A. It is clear from (3.1) and (3.2) that the open dense set $\mathcal{A}$ is plurithin at $0 \in E^{n}$. By Proposition 3.1, the closed set $E^{n} \backslash \mathcal{A}$ does not separate domains. Therefore this set cannot contain any open subset of a real hypersurface. Thus $\mathcal{A}$ has all the desired properties.

Proof of Proposition 3.1. One first observes that

$$
\mathcal{S} \subset E^{n} \backslash \mathcal{A}=\left\{z \in E^{n}: u(z) \geq-1\right\} .
$$

For any four vectors $a:=\left(a_{1}, \ldots, a_{n}\right), b:=\left(b_{1}, \ldots, b_{n}\right), c:=\left(c_{1}, \ldots, c_{n}\right)$, $d:=\left(d_{1}, \ldots, d_{n}\right)$ in $\mathbb{R}^{n}$ with the property that $a_{k}<b_{k}$ and $c_{k}<d_{k}, k=$ $1, \ldots, n$, one defines an open cube in $\mathbb{C}^{n}$ by

$$
\begin{aligned}
\Delta & =\Delta(a, b, c, d) \\
& :=\left\{z \in \mathbb{C}^{n}: a_{k}<\operatorname{Re} z_{k}<b_{k}, c_{k}<\operatorname{Im} z_{k}<d_{k}, k=1, \ldots, n\right\} .
\end{aligned}
$$

It is clear that the intersection of two such cubes is either empty or a cube.

One first shows that for any cube $\Delta \subset E^{n}$ the open set $\Delta \backslash \mathcal{S}$ is connected. Indeed, pick two points $z=\left(z_{1}, \ldots, z_{n}\right)$ and $w=\left(w_{1}, \ldots, w_{n}\right)$ in $\Delta \backslash \mathcal{S}$. Since $\{z \in E: v(z)=-\infty\}$ is dense in $E$, we can choose $z^{\prime}=\left(z_{1}^{\prime}, \ldots, z_{n}^{\prime}\right)$ and $w^{\prime}=\left(w_{1}^{\prime}, \ldots, w_{n}^{\prime}\right)$ in $\Delta \backslash \mathcal{S}$ such that 
(i) the segments $\gamma_{1}(t):=(1-t) z+t z^{\prime}$ and $\gamma_{3}(t):=(1-t) w+t w^{\prime}$, $0 \leq t \leq 1$, are contained in $\Delta \backslash \mathcal{S}$;

(ii) $z_{1}^{\prime}, \ldots, z_{n}^{\prime}$ and $w_{1}^{\prime}, \ldots, w_{n}^{\prime}$ are in $\{z \in E: v(z)=-\infty\}$.

Consider now $\gamma_{2}:[0,1] \rightarrow \Delta$ given by

$$
\gamma_{2}(t):=\left(w_{1}^{\prime}, \ldots, w_{j}^{\prime},(j+1-n t) z_{j+1}^{\prime}+(n t-j) w_{j+1}^{\prime}, z_{j+2}^{\prime}, \ldots, z_{n}^{\prime}\right)
$$

for $t \in[j / n,(j+1) / n]$ and $j=0, \ldots, n-1$. By (3.2) and property (ii) above, $\gamma_{2}(t) \in\left\{z \in E^{n}: u(z)=-\infty\right\}$ for $t \in[0,1]$. This implies that $\gamma_{2}:[0,1] \rightarrow \Delta \backslash \mathcal{S}$.

Observe that $\gamma_{2}(0)=z^{\prime}$ and $\gamma_{2}(1)=w^{\prime}$. By (i), the new path $\gamma:[0,1] \rightarrow$ $\Delta \backslash \mathcal{S}$ given by

$$
\gamma(t):= \begin{cases}\gamma_{1}(3 t), & t \in[0,1 / 3], \\ \gamma_{2}(3 t-1), & t \in[1 / 3,2 / 3], \\ \gamma_{3}(3 t-2), & t \in[2 / 3,1],\end{cases}
$$

satisfies $\gamma(0)=z$ and $\gamma(1)=w$, and $\Delta \backslash \mathcal{S}$ is therefore connected.

Now let $U$ be any subdomain of $E^{n}$. We wish to show that $U \backslash \mathcal{S}$ is connected. To do this, pick points $z=\left(z_{1}, \ldots, z_{n}\right)$ and $w=\left(w_{1}, \ldots, w_{n}\right)$ in $U \backslash \mathcal{S}$. Since $U$ is arcwise connected, there is a continuous function $\gamma$ : $[0,1] \rightarrow U$ such that $\gamma(0)=z$ and $\gamma(1)=w$.

By the Heine-Borel Theorem, the compact set $\mathcal{L}:=\gamma([0,1])$ can be covered by a finite number of cubes $\Delta_{l}(1 \leq l \leq N)$ with $\Delta_{l} \subset U$ and $\Delta_{l} \cap \mathcal{L} \neq \emptyset$. Since the path $\mathcal{L}$ is connected, the union $\bigcup_{l=1}^{N} \Delta_{l}$ is also connected.

Suppose without loss of generality that $z \in \Delta_{1}$ and $w \in \Delta_{N}$. From the discussion above, if $\Delta_{1} \cap \Delta_{2} \neq \emptyset$ then $\left(\Delta_{1} \backslash \mathcal{S}\right) \cap\left(\Delta_{2} \backslash \mathcal{S}\right)=\left(\Delta_{1} \cap \Delta_{2}\right) \backslash \mathcal{S}$ is connected, and hence $\left(\Delta_{1} \cup \Delta_{2}\right) \backslash \mathcal{S}$ is also connected. Repeating this argument at most $N$ times and using the connectivity of $\bigcup_{l=1}^{N} \Delta_{l}$, we finally conclude that $\bigcup_{l=1}^{N} \Delta_{l} \backslash \mathcal{S}(\subset U \backslash \mathcal{S})$ is also connected. This completes the proof.

COROLlary 3.2. (i) If $\mathcal{S}_{1}, \ldots, \mathcal{S}_{N}$ are relatively closed subsets of $E^{n}$ which do not separate domains, then the union $\bigcup_{l=1}^{N} \mathcal{S}_{l}$ does not separate domains either.

(ii) Let $\mathcal{A}, \mathcal{S}$ be as in Proposition 3.1. Then for any closed sets $F_{1}$ in $\mathbb{C}^{p}$ and $F_{2}$ in $\mathbb{C}^{q}(p, q \geq 0)$, the closed set $F_{1} \times \mathcal{S} \times F_{2}$ does not separate domains in $\mathbb{C}^{p} \times E^{n} \times \mathbb{C}^{q}$.

Proof. To prove (i), let $U$ be any subdomain of $E^{n}$. Since $U \backslash\left(\bigcup_{l=1}^{N} \mathcal{S}_{l}\right)=$ $\left(\ldots\left(U \backslash \mathcal{S}_{1}\right) \ldots \backslash \mathcal{S}_{N}\right)$, part (i) follows from the hypothesis on the $\mathcal{S}_{l}$ 's.

To prove part (ii), consider any subdomain $U$ of $\mathbb{C}^{p} \times E^{n} \times \mathbb{C}^{q}$ and let $\left(z_{1}, w_{1}, t_{1}\right),\left(z_{2}, w_{2}, t_{2}\right)$ be two points in $U \backslash\left(F_{1} \times \mathcal{S} \times F_{2}\right)$. Since $\mathcal{A}$ is an open dense subset of $E^{n}, \operatorname{int}\left(F_{1} \times \mathcal{S} \times F_{2}\right)=\emptyset$, and therefore we can employ the 
compactness argument that we already used in the proof of Proposition 3.1. Consequently, one is reduced to the case where $U$ is a cube in $\mathbb{C}^{p+n+q}$.

Another reduction is in order. Since $U \backslash\left(F_{1} \times \mathcal{S} \times F_{2}\right)$ is open and $\mathcal{A}$ is dense in $E^{n}$, by replacing $w_{1}$ (resp. $w_{2}$ ) by $w_{1}^{\prime}$ (resp. $w_{2}^{\prime}$ ) close to $w_{1}$ (resp. $w_{2}$ ), we may suppose that $w_{1}, w_{2} \in E^{n} \backslash \mathcal{S}$.

Write the cube $U$ as the product $\Delta_{1} \times \Delta_{2} \times \Delta_{3}$, where $\Delta_{1}$ (resp. $\Delta_{2}$ and $\left.\Delta_{3}\right)$ is a cube in $\mathbb{C}^{p}$ (resp. $\mathbb{C}^{n}$ and $\mathbb{C}^{q}$ ). By Proposition 3.1 , there is a continuous path $\gamma_{2}:[0,1] \rightarrow \Delta_{2} \backslash \mathcal{S}$ such that $\gamma_{2}(0)=w_{1}$ and $\gamma_{2}(1)=w_{2}$.

We now consider the path $\gamma:[0,1] \rightarrow \Delta_{1} \times \Delta_{2} \times \Delta_{3} \backslash \mathcal{S}$ where $\gamma(t):=$ $\left(\gamma_{1}(t), \gamma_{2}(t), \gamma_{3}(t)\right)$ and $\gamma_{1}(t):=(1-t) z_{1}+t z_{2}, \gamma_{3}(t):=(1-t) t_{1}+t t_{2}$, $t \in[0,1]$. It is easy to see that $\gamma(0)=\left(z_{1}, w_{1}, t_{1}\right)$ and $\gamma(1)=\left(z_{2}, w_{2}, t_{2}\right)$, which finishes the proof.

The following two lemmas will be crucial for the proof of Propositions B and $\mathrm{C}$.

LEMMA 3.3. For an open set $\Omega \subset \mathbb{C}^{n}$ and $A \subset \Omega$, we have either $\omega_{A, \Omega} \equiv 0$ or $\sup _{\Omega} \omega_{A, \Omega}=1$.

Proof. We first prove the lemma in the case where $\Omega$ is bounded. Suppose in order to get a contradiction that $\sup _{\Omega} h_{A, \Omega}^{*}=M$ with $0<M<1$. By the definition of $h_{A, \Omega}^{*}$, it follows that

$$
\begin{aligned}
\{u: u \in \operatorname{PSH}(\Omega), u & \leq 1 \text { on } \Omega, u \leq 0 \text { on } A\} \\
& =\{u: u \in \operatorname{PSH}(\Omega), u \leq M \text { on } \Omega, u \leq 0 \text { on } A\} .
\end{aligned}
$$

Therefore, $h_{A, \Omega}^{*}(z) \leq M h_{A, \Omega}^{*}<h_{A, \Omega}^{*}$ for any $z \in \Omega$ with $h_{A, \Omega}^{*}(z)>0$, and we obtain the desired contradiction.

The general case is analogous using the definition of $\omega_{A, \Omega}$.

LEMMA 3.4. Let $\Omega_{1} \subsetneq \Omega_{2}$ be two domains of $\mathbb{C}^{n}$ such that $\Omega_{2}$ is pseudoconvex. Assume that there is an upper bounded function $\phi \in \operatorname{PSH}\left(\Omega_{2}\right)$ satisfying $\Omega_{1}=\left\{z \in \Omega_{2}: \phi(z)<0\right\}$. Then there is a function $f \in \mathcal{O}\left(\Omega_{1}\right)$ such that there is no function $\widehat{f} \in \mathcal{M}\left(\Omega_{2}\right)$ with $\widehat{f}=f$ on $\Omega_{1}$.

Proof. It is clear from the hypothesis that $\Omega_{1}$ is also pseudoconvex. Let $\partial \Omega_{1}$ be the boundary of $\Omega_{1}$ in $\Omega_{2}$ and let $S$ be a countable dense subset of $\partial \Omega_{1}$. It is a classical fact that there is a function $f \in \mathcal{O}\left(\Omega_{1}\right)$ such that

$$
\lim _{z \in \Omega_{1}, z \rightarrow w}|f(z)|=\infty, \quad w \in S .
$$

We will show that this is the desired function. Indeed, suppose in order to get a contradiction that there is a function $\widehat{f} \in \mathcal{M}\left(\Omega_{2}\right)$ satisfying $\widehat{f}=f$ on $\Omega_{1}$. Because of (3.3), $S \cap \Omega_{2}$ and then $\partial \Omega_{1} \cap \Omega_{2}$ are contained in the pole set of $\widehat{f}$ (i.e. the union of the set of all poles of $\widehat{f}$ and the set of all indeterminacy points of $\widehat{f}$ ). Therefore, for any point $w \in \partial \Omega_{1} \cap \Omega_{2}$, there is a small open neighborhood $U$ of $w$ and a complex analytic subset $C$ 
of codimension one such that $U \backslash C \subset \Omega_{1}$. Since $\phi \in \operatorname{PSH}(U)$ is upper bounded, $\phi(w)=\limsup _{z \in U \backslash C, z \rightarrow w} \phi(z)=\phi(w) \leq 0$ for all $w \in \partial \Omega_{1}$. Since $\Omega_{1} \subsetneq \Omega_{2}, \phi$ is non-constant and therefore $\phi(w)<0$ for all $w \in \partial \Omega_{1}$, which is a contradiction.

We are now ready to prove Propositions B and C.

Proof of Proposition B. Suppose, without loss of generality, that $D=E^{p}$ and $G=E^{q}$. The general case is analogous. Let $F_{p}$ (resp. $F_{q}$ ) be any closed ball contained in the open set $\mathcal{A}_{p}$ (resp. $\mathcal{A}_{q}$ ). Define the relatively closed set $S$ by

$$
S:=\left(E^{p} \backslash \mathcal{A}_{p}\right) \times F_{q} \cup F_{p} \times\left(E^{q} \backslash \mathcal{A}_{q}\right) .
$$

We now check the properties (i) and (ii) of Proposition B. First, int $S=\emptyset$ because $\mathcal{A}_{p}$ (resp. $\mathcal{A}_{q}$ ) is open dense set in $E^{p}$ (resp. $E^{q}$ ). Second, by Proposition 3.1 and Corollary 3.2(ii), the two relatively closed sets $\left(E^{p} \backslash \mathcal{A}_{p}\right)$ $\times F_{q}$ and $F_{p} \times\left(E^{q} \backslash \mathcal{A}_{q}\right)$ do not separate domains. By Corollary 3.2(i), the union $S$ also enjoys this property. Thus $S$ satisfies (i).

Using (3.4), a direct computation entails that $A=\mathcal{A}_{p}$ and $B=\mathcal{A}_{q}$ and $A, B$ are open, in particular they are locally pluriregular.

By the classical Cross Theorem (see for instance [7] or [1]), the envelope of holomorphy of $X$ is given by

$$
\widehat{X}:=\left\{(z, w) \in E^{p} \times E^{q}: \omega_{A, E^{p}}(z)+\omega_{B, E^{q}}(w)<1\right\} .
$$

We now show that $h_{\mathcal{A}_{n}, E^{n}}^{*}(0)>0$ for $n \geq 2$. Indeed, let $M:=\sup _{E^{n}} u$, where $u$ is defined in (3.2). Observe that $M>0$ since $u(0)=0$. Consider the function $\widetilde{u} \in \operatorname{PSH}\left(E^{n}\right)$ given by

$$
\widetilde{u}(z):=\frac{u(z)-M}{M+\frac{1}{2}}+1 \quad \text { for } z \in E^{n} .
$$

It can be easily checked that $\widetilde{u}(z) \leq 1$ on $E^{n}$ and $\widetilde{u}(z) \leq 0$ on $\mathcal{A}_{n}$. Thus $\widetilde{u}(0) \leq h_{\mathcal{A}_{n}, E^{n}}^{*}(0)$. On the other hand, $\widetilde{u}(0)=1 /(2 M+1)>0$. Hence our assertion above follows.

We next show that $\widehat{X} \subsetneq E^{p} \times E^{q}$. Indeed, we have

$$
\left\{w \in E^{q}:(0, w) \in \widehat{X}\right\} \subset\left\{w \in E^{q}: h_{\mathcal{A}_{q}, E^{q}}^{*}(w)<1-h_{\mathcal{A}_{p}, E^{p}}^{*}(0)\right\} .
$$

Since $h_{\mathcal{A}_{q}, E^{q}}^{*}(0)>0$ and $h_{\mathcal{A}_{p}, E^{p}}^{*}(0)>0$, Lemma 3.3 applies, and consequently, the latter set is strictly contained in $E^{q}$. This proves our assertion.

We are now ready to complete the proof. By Lemma 3.4, there is a holomorphic function $f$ in $\widehat{X}$ which cannot be meromorphically extended to $E^{p} \times E^{q}$. Therefore, there is no meromorphic function $\widehat{f} \in \mathcal{M}\left(E^{p} \times E^{q}\right)$ such that $\widehat{f}=f$ on

$$
X \backslash S=\left(\left(\mathcal{A}_{p} \backslash F_{p}\right) \times E^{q}\right) \cup\left(\mathcal{A}_{p} \times \mathcal{A}_{q}\right) \cup\left(E^{p} \times\left(\mathcal{A}_{q} \backslash F_{q}\right)\right),
$$


which is a set of unicity for meromorphic functions. The proof is thereby finished.

Proof of Proposition $C$. In order to simplify the notation, we only consider the case $n=3$, the general case $n>3$ is analogous. Let $\mathcal{B}$ be the following open dense subset of $E$ :

$$
\mathcal{B}:=\{z \in E: v(z)<-1 / 2\}
$$

where $v$ is given by (3.1). Then by virtue of (3.2), it can be checked that $(E \backslash \mathcal{B}) \times(E \backslash \mathcal{B}) \subset E^{2} \backslash \mathcal{A}_{2}$. Fix any closed ball $F$ contained in the open set $\mathcal{B}$. Next apply Proposition 3.1 and Corollary 3.2 to the relatively closed set $\mathcal{S}:=(E \backslash \mathcal{B}) \times(E \backslash \mathcal{B})$. Consequently, the set

$$
\begin{aligned}
S:= & (F \times(E \backslash \mathcal{B}) \times(E \backslash \mathcal{B})) \cup((E \backslash \mathcal{B}) \times F \times(E \backslash \mathcal{B})) \\
& \cup((E \backslash \mathcal{B}) \times(E \backslash \mathcal{B}) \times F)
\end{aligned}
$$

does not separate domains in $E^{3}$. Moreover, since $\mathcal{B}$ is an open dense subset of $E$, we see that int $S=\emptyset$ and $S$ is relatively closed. Hence $S$ satisfies property (i).

To verify (ii), one first computes the following sets using (3.5):

$$
\begin{aligned}
S_{1} & =S_{2}=S_{3}=(E \backslash \mathcal{B}) \times(E \backslash \mathcal{B}), \\
T & =(\mathcal{B} \times E \times E) \cup(E \times \mathcal{B} \times E) \cup(E \times E \times \mathcal{B}) .
\end{aligned}
$$

Next, by the product property for the relative extremal function [6], we have $h_{\mathcal{B} \times \mathcal{B}, E^{2}}^{*}(0)=h_{\mathcal{B}, E}^{*}(0)$. Since $\mathcal{B} \times \mathcal{B} \subset \mathcal{A}_{2}$ and we have shown in Proposition $\mathrm{B}$ that $h_{\mathcal{A}_{2}, E^{2}}^{*}(0)>0$, it follows that $h_{\mathcal{B}, E}^{*}(0)>0$.

Consider now the domain of holomorphy

$$
\Omega:=\left\{(z, w, t) \in E^{3}: h_{\mathcal{B}, E}^{*}(z)+h_{\mathcal{B}, E}^{*}(w)+h_{\mathcal{B}, E}^{*}(t)<2\right\} .
$$

Since $\mathcal{B}$ is open and therefore locally pluriregular, it can be proved using Lemma 5 in [2] that $\Omega$ is a domain. Moreover it can be easily checked that $T \subset \Omega$ using (3.6) and (3.7).

We now prove that $\Omega \subsetneq E^{3}$. Indeed, since $h_{\mathcal{B}, E}^{*}(0)>0$, by Lemma 3.3 there are $z, w \in E$ such that $h_{\mathcal{B}, E}^{*}(z)>2 / 3$ and $h_{\mathcal{B}, E}^{*}(w)>2 / 3$. Then the fiber

$$
\{t \in E:(z, w, t) \in \Omega\} \subset\left\{t \in E: h_{\mathcal{B}, E}^{*}(t)<2 / 3\right\} .
$$

Another application of Lemma 3.3 shows that the latter set is strictly contained in $E$. This proves our assertion.

We can now complete the proof. By Lemma 3.4, there is a holomorphic function $f$ in $\Omega$ which cannot be meromorphically extended to $E^{n}$. Therefore, there is no meromorphic function $\widehat{f} \in \mathcal{M}\left(E^{n}\right)$ such that $\widehat{f}=f$ on $T \backslash S$, a set of unicity for meromorphic functions. Hence, the proof is finished. 
4. Auxiliary results. Let $S$ be a subset of an open set $D \subset \mathbb{C}^{n}$. Then $S$ is said to be of Baire category $I$ if $S$ is contained in a countable union of relatively closed sets in $D$ with empty interior. Otherwise, $S$ is said to be of Baire category II.

The following lemma is very useful.

Lemma 4.1. For $j \in\{1, \ldots, M\}$ and $M \geq 2$, let $\Omega_{j}$ be a domain in $\mathbb{C}^{m_{j}}$ and let $S$ be a relatively closed subset of $\Omega_{1} \times \ldots \times \Omega_{M}$ with $\operatorname{int} S=\emptyset$. For $a_{j} \in \Omega_{j}, j \in\{3, \ldots, M\}$, let $S\left(a_{3}, \ldots, a_{M}\right)$ denote the set of all $a_{2} \in \Omega_{2}$ such that $\operatorname{int}_{\mathbb{C}^{m_{1}}} S\left(\cdot, a_{2}, a_{3}, \ldots, a_{M}\right)=\emptyset$. For $j \in\{4, \ldots, M\}$, let $S\left(a_{j}, \ldots, a_{M}\right)$ denote the set of all $a_{j-1} \in \Omega_{j-1}$ such that $\Omega_{j-2} \backslash S\left(a_{j-1}, a_{j}, \ldots, a_{M}\right)$ is of Baire category $I$, and finally let $\mathcal{S}$ denote the set of all $a_{M} \in \Omega_{M}$ such that $\Omega_{M-1} \backslash S\left(a_{M}\right)$ is of Baire category I. Then $\Omega_{M} \backslash \mathcal{S}$ is of Baire category I.

Proof. For $j \in\{1, \ldots, M\}$ let $(\mathbb{Q}+i \mathbb{Q})^{m_{j}}=\left\{q_{1}^{j}, \ldots, q_{n}^{j}, \ldots\right\}$ and $\delta_{n}:=$ $1 / n, n \in \mathbb{N}$. For $q \in \Omega_{j}$ and $r>0$, let $\Delta_{q}(r)$ denote the polydisc in $\mathbb{C}^{m_{j}}$ with center $q$ and multi-radius $(r, \ldots, r)$.

Suppose to get a contradiction that $\Omega_{M} \backslash \mathcal{S}$ is of Baire category II. Then for all $a_{M} \in \Omega_{M} \backslash \mathcal{S}, \Omega_{M-1} \backslash S\left(a_{M}\right)$ is of Baire category II. Therefore, for $j=$ $M-1, \ldots, 3$ and any $a_{j} \in \Omega_{j} \backslash S\left(a_{j+1}, \ldots, a_{M}\right)$, the set $\Omega_{j-1} \backslash S\left(a_{j}, \ldots, a_{M}\right)$ is of Baire category II. Put

$$
S_{n}\left(a_{3}, \ldots, a_{M}\right):=\left\{a_{2} \in \Omega_{2}: S\left(\cdot, a_{2}, a_{3}, \ldots, a_{M}\right) \supset \Delta_{q_{n}^{1}}\left(\delta_{n}\right)\right\} .
$$

Since $S$ is relatively closed, $S_{n}\left(a_{3}, \ldots, a_{M}\right)$ is also relatively closed in $\Omega_{2}$. Moreover, from the definition of $S\left(a_{3}, \ldots, a_{M}\right)$, we have the identity

$$
\Omega_{2} \backslash S\left(a_{3}, \ldots, a_{M}\right)=\bigcup_{n=1}^{\infty} S_{n}\left(a_{3}, \ldots, a_{M}\right) .
$$

Since it is shown in the above discussion that $\Omega_{2} \backslash S\left(a_{3}, \ldots, a_{M}\right)$ is of Baire category II in $\Omega_{2}$, we can apply the Baire Theorem to the right side of the latter identity. Consequently, there exist $n_{1}, n_{2} \in \mathbb{N}$ such that $S_{n_{1}}\left(a_{3}, \ldots, a_{M}\right) \supset \Delta_{q_{n_{2}}^{2}}\left(\delta_{n_{2}}\right)$. This implies that $S\left(\cdot, \cdot, a_{3}, \ldots, a_{M}\right) \supset$ $\Delta_{q_{n_{1}}^{1}}\left(\delta_{n_{1}}\right) \times \Delta_{q_{n_{2}}^{2}}\left(\delta_{n_{2}}\right)$.

Now define inductively, for $j=2, \ldots, M-1$ and $n_{1}, \ldots, n_{j} \in \mathbb{N}$,

$$
\begin{aligned}
& S_{n_{1}, \ldots, n_{j}}\left(a_{j+2}, \ldots, a_{M}\right) \\
& \quad:=\left\{a_{j+1} \in \Omega_{j+1}: S\left(\ldots, a_{j+1}, \ldots, a_{M}\right) \supset \Delta_{q_{n_{1}}^{1}}\left(\delta_{n_{1}}\right) \times \ldots \times \Delta_{q_{n_{j}}^{j}}\left(\delta_{n_{j}}\right)\right\} .
\end{aligned}
$$

Since $S$ is relatively closed, $S_{n_{1}, \ldots, n_{j}}\left(a_{j+2}, \ldots, a_{M}\right)$ is also relatively closed. Moreover, it can be checked that

$$
\Omega_{j+1} \backslash S\left(a_{j+2}, \ldots, a_{M}\right) \subset \bigcup_{n_{1}, \ldots, n_{j}=1}^{\infty} S_{n_{1}, \ldots, n_{j}}\left(a_{j+2}, \ldots, a_{M}\right) .
$$


By the Baire Theorem again, it follows that there are $n_{1}, \ldots, n_{j+1} \in \mathbb{N}$ such that $S_{n_{1}, \ldots, n_{j}}\left(a_{j+2}, \ldots, a_{M}\right) \supset \Delta_{q_{n_{j+1}^{j+1}}}\left(\delta_{n_{j+1}}\right)$, and hence

$$
S\left(\ldots, a_{j+2}, \ldots, a_{M}\right) \supset \Delta_{q_{n_{1}}^{1}}\left(\delta_{n_{1}}\right) \times \ldots \times \Delta_{q_{n_{j+1}+1}^{j}}\left(\delta_{n_{j+1}}\right) .
$$

Finally, we deduce for $j=M-1$ that int $S \neq \emptyset$, which contradicts the hypothesis. Hence, the proof is complete.

REMARK 4.2. If we apply Lemma 4.1 to the case where $\Omega_{1}:=D_{j}$ and $\Omega_{2}:=\left(D_{1} \times \ldots \times D_{j-1}\right) \times\left(D_{j+1} \times \ldots \times D_{N}\right)$ then, for each $j \in\{1, \ldots, N\}$, the set $S_{j}$ in the statement of Theorem D is of Baire category I. In particular, the set $\Omega \backslash((T \backslash S) \cap \Omega)$ is of Baire category I for all open sets $\Omega \subset D$.

Lemma 4.3. Let $U \subset \mathbb{C}^{p}$ and $V \subset \mathbb{C}^{q}$ be two pseudoconvex domains. Consider four sets $C \subset A \subset U$ and $D \subset B \subset V$ such that $\bar{C}=\bar{A}, \bar{D}=$ $\bar{B}$ and $\bar{A}, \bar{B}$ are locally pluriregular. Put $X:=\mathbb{X}(A, B ; U, V)$ and $\widehat{X}:=$ $\widehat{\mathbb{X}}(\bar{A}, \bar{B} ; U, V)$. Assume $f \in \mathcal{O}_{\mathrm{S}}(X)$ and there is a finite constant $K$ such that for all $c \in C$ and $d \in D$,

$$
\sup _{V}|f(c, \cdot)|<K \quad \text { and } \quad \sup _{U}|f(\cdot, d)|<K
$$

Then there exists a unique function $\widehat{f} \in \mathcal{O}(\widehat{X})$ such that $\widehat{f}=f$ on $X$.

Proof. From the hypothesis on the boundedness of $f$, it follows that the two families $\{f(c, \cdot): c \in C\}$ and $\{f(\cdot, d): d \in D\}$ are normal. We now define two functions $f_{1}$ on $\bar{A} \times V$ and $f_{2}$ on $U \times \bar{B}$ as follows.

For any $z \in \bar{A}$, choose a sequence $\left(c_{n}\right)_{n=1}^{\infty} \subset C$ such that $\lim _{n \rightarrow \infty} c_{n}=z$ and the sequence $\left(f\left(c_{n}, \cdot\right)\right)_{n=1}^{\infty}$ converges uniformly on compact subsets of $V$. We let

$$
f_{1}(z, w):=\lim _{n \rightarrow \infty} f\left(c_{n}, w\right) \quad \text { for all } w \in V
$$

Similarly, for any $w \in \bar{B}$, choose a sequence $\left(d_{n}\right)_{n=1}^{\infty} \subset D$ such that $\lim _{n \rightarrow \infty} d_{n}$ $=w$ and the sequence $\left(f\left(\cdot, d_{n}\right)\right)_{n=1}^{\infty}$ converges uniformly on compact subsets of $U$. We let

$$
f_{2}(z, w):=\lim _{n \rightarrow \infty} f\left(z, d_{n}\right) \quad \text { for all } z \in U .
$$

We first check that $f_{1}$ and $f_{2}$ are well defined. Indeed, it suffices to verify this for $f_{1}$ since the same argument also applies to $f_{2}$. Let $\left(c_{n}^{\prime}\right)_{n=1}^{\infty} \subset C$ be another sequence such that $\lim _{n \rightarrow \infty} c_{n}^{\prime}=z$ and the sequence $\left(f\left(c_{n}^{\prime}, \cdot\right)\right)_{n=1}^{\infty}$ converges uniformly on compact subsets of $V$. Since for all $b \in B$,

$$
\lim _{n \rightarrow \infty} f\left(c_{n}, b\right)=f(z, b)=\lim _{n \rightarrow \infty} f\left(c_{n}^{\prime}, b\right),
$$

and since $B$ is a set of unicity for holomorphic functions on $V$, our claim follows. 
One next verifies that $f_{1}=f_{2}$ on $\bar{A} \times \bar{B}$. Indeed, let $z \in \bar{A}, w \in \bar{B}$ and let $\left(c_{n}\right)_{n=1}^{\infty} \subset C,\left(d_{n}\right)_{n=1}^{\infty} \subset D$ be as above. Then clearly, we have

$$
f_{1}(z, w)=\lim _{n \rightarrow \infty} f\left(c_{n}, d_{n}\right)=f_{2}(z, w) .
$$

We are now able to define a function $\widetilde{f}$ on $\mathbb{X}(\bar{A}, \bar{B} ; U, V)$ by $f=f_{1}$ on $\bar{A} \times V$ and $f=f_{2}$ on $U \times \bar{B}$. It follows from the construction of $f_{1}$ and $f_{2}$ that $\widetilde{f} \in \mathcal{O}_{\mathrm{s}}(\mathbb{X}(\bar{A}, \bar{B} ; U, V))$.

One next checks that $\tilde{f}=f$ on $X$. Indeed, since for each $a \in A, f(a, \cdot)$ and $\widetilde{f}(a, \cdot)$ are holomorphic, it suffices to verify that $\widetilde{f}(a, d)=f(a, d)$. But the latter equality follows easily from the definition of $f_{1}$ and the hypothesis.

Finally, one applies the classical Cross Theorem (see for instance [7], [1]) to $\tilde{f} \in \mathcal{O}_{\mathrm{s}}(\mathbb{X}(\bar{A}, \bar{B} ; U, V))$; thus the existence of $\widehat{f}$ follows. The unicity of $\widehat{f}$ is also clear.

Lemma 4.4 (Rothstein type theorem, cf. [8]). Let $f \in \mathcal{O}\left(E^{p} \times E^{q}\right)$. Assume that $A \subset E^{p}$ is such that for all open subsets $U \subset E^{p}, A \cap U$ is of Baire category II. Let $G \subset \mathbb{C}^{q}$ be a domain such that $E^{q} \subset G$ and assume that for all $a \in A$, the function $f(a, \cdot)$ extends meromorphically to $\widetilde{f}(a, \cdot) \in \mathcal{M}(G)$. Then for any relatively compact subdomain $\widetilde{G} \subset G$, there are an open dense set $\mathcal{A} \subset E^{p}$ and a function $\widetilde{f} \in \mathcal{M}(\Omega)$, where $\Omega:=E^{p} \times E^{q} \cup \mathcal{A} \times \widetilde{G}$, such that $\widetilde{f}=f$ on $E^{p} \times E^{q}$.

Sketch of proof. (1) The case where $G:=\Delta_{0}(R)(R>1)$. Argue as in the proof of Rothstein's theorem given in [10].

(2) The general case. Fix an $a \in E^{p}$ and $r>0$. Let $B$ denote the set of all $b \in G$ such that there exist $0<r_{b}<r$, an open dense subset $A_{b}$ of $\Delta_{a}\left(r_{b}\right)$ and $f_{b} \in \mathcal{M}\left(A_{b} \times \Delta_{b}\left(r_{b}\right)\right)$ such that $f_{b}(\alpha, \cdot)=\widetilde{f}(\alpha, \cdot)$ on $\Delta_{b}\left(r_{b}\right)$ for all $\alpha \in A \cap A_{b}$.

Obviously, $B$ is open. Using case (1) and the hypothesis on $A$, one can show that $B$ is closed in $G$. Thus $B=G$. Moreover, one can also show that if $A_{b} \cap A_{b^{\prime}} \neq \emptyset$ and $\Delta_{b}\left(r_{b}\right) \cap \Delta_{b^{\prime}}\left(r_{b^{\prime}}\right) \neq \emptyset$, then $f_{b}=f_{b^{\prime}}$ on $\left(A_{b} \cap A_{b^{\prime}}\right) \times\left(\Delta_{b}\left(r_{b}\right) \cap\right.$ $\left.\Delta_{b^{\prime}}\left(r_{b^{\prime}}\right)\right)$. Therefore, using the hypothesis that $\widetilde{G}$ is relatively compact, we see that for any $a \in E^{p}$ and any $r>0$, there is an open set $\mathcal{A}_{a, r} \subset \Delta_{a}(r)$ and $f_{a, r} \in \mathcal{M}\left(\mathcal{A}_{a, r} \times \widetilde{G}\right)$ such that $f_{a, r}(\alpha, \cdot)=\widetilde{f}(\alpha, \cdot)$ on $\widetilde{G}$ for all $\alpha \in A \cap \mathcal{A}_{a, r}$.

Finally, let $\mathcal{A}:=\bigcup_{a \in E^{n}, r>0} \mathcal{A}_{a, r}$. This open set is clearly dense in $E^{p}$. By gluing the functions $f_{a, r}$ together, we obtain the desired meromorphic extension $\tilde{f} \in \mathcal{M}(\Omega)$; so the proof of the lemma is complete.

5. Proof of Theorem D. We will only give the proof of Theorem D for the case where $f$ is separately meromorphic. Since the case where $f$ is separately holomorphic is quite similar and in some sense simpler, it is left to the reader. 
Proof of (ii). Put

$$
\mathcal{A}_{j}:=\left\{z_{j} \in D_{j}: \operatorname{int}_{\mathbb{C}^{n_{j}}} S\left(z_{j}, \cdot\right)=\emptyset\right\} \quad \text { for } j=1,2 .
$$

By Lemma 4.1, $D_{j} \backslash \mathcal{A}_{j}$ is of Baire category I. For $a_{j} \in \mathcal{A}_{j}(j=1,2)$, let $\widetilde{f\left(a_{1}, \cdot\right)}$ (resp. $\left.\widetilde{f\left(\cdot, a_{2}\right)}\right)$ denote the meromorphic extension of $f\left(a_{1}, \cdot\right)$ (resp. $\left.f\left(\cdot, a_{2}\right)\right)$ to $D_{2}$ (resp. to $D_{1}$ ).

Let $\mathcal{U} \subset D_{1}, \mathcal{V} \subset D_{2}$ be arbitrary open sets. For a relatively compact pseudoconvex subdomain $V$ of $\mathcal{V}$ and for a positive number $K$, let $Q_{V, K}^{1}$ denote the set of $a_{1} \in \mathcal{A}_{1} \cap \mathcal{U}$ such that $\sup _{V}\left|\widetilde{f\left(a_{1}, \cdot\right)}\right| \leq K$ (and thus $\left.\widetilde{f\left(a_{1}, \cdot\right)} \in \mathcal{O}(V)\right)$. By $(5.1)$ and the hypothesis, a countable number of the $Q_{V, K}^{1}$ cover $\mathcal{A}_{1} \cap \mathcal{U}$. Since the latter set is of Baire category II, we can choose $V, K_{1}$ such that the closure $\overline{Q_{V, K_{1}}^{1}}$ contains a polydisc $\Delta_{1} \subset \mathcal{U}$ and $Q_{V, K_{1}}^{1} \cap \Delta_{1}$ is of Baire category II in $\Delta_{1}$.

For a relatively compact pseudoconvex subdomain $U$ of $\Delta_{1}$ and for a positive number $K$, we denote by $Q_{U, K}^{2}$ the set of $a_{2} \in \mathcal{A}_{2} \cap V$ such that $\sup _{U}\left|\widetilde{f\left(\cdot, a_{2}\right)}\right| \leq K$ (and thus $\left.\widetilde{f\left(\cdot, a_{2}\right)} \in \mathcal{O}(U)\right)$. By (5.1) and the hypothesis, a countable number of the $Q_{U, K}^{2}$ cover $\mathcal{A}_{2} \cap V$. Since the latter set is of Baire category II, we can choose $U, K_{2}$ such that $\overline{Q_{U, K_{2}}^{2}}$ contains a polydisc $\Delta_{2} \subset V$ and $Q_{U, K_{2}}^{2} \cap \Delta_{2}$ is of Baire category II in $\Delta_{2}$.

Now let $K:=\max \left\{K_{1}, K_{2}\right\}, A:=\mathcal{A}_{1} \cap U, C:=Q_{V, K}^{1} \cap U, B:=\mathcal{A}_{2} \cap \Delta_{2}$, $D:=Q_{V, K}^{2} \cap \Delta_{2}$. Then it is easy to see that $\bar{A}=\bar{C}=U$ and $\bar{B}=\bar{D}=\Delta_{2}$. Moreover, all other hypotheses of Lemma 4.3 are satisfied. Consequently, an application of this lemma gives the following.

Let $\mathcal{U} \subset D_{1}, \mathcal{V} \subset D_{2}$ be arbitrary open sets. Then there are a polydisc $\Delta_{a}(r) \subset \mathcal{U} \times \mathcal{V}$ and a function $\widehat{f} \in \mathcal{O}\left(\Delta_{a}(r)\right)$ such that $\widehat{f}=f$ on $(T \backslash S) \cap$ $\Delta_{a}(r)$.

Write $a=\left(a_{1}, a_{2}\right) \in D_{1} \times D_{2}$. Since the set $\mathcal{A}_{j} \cap \Delta_{a_{j}}(r)$ is of Baire category II, by replacing $\Delta_{a_{j}}(r)$ with a smaller polydisc we see that this set satisfies the hypothesis of Lemma 4.4. Consequently, an application of that lemma gives $f_{a}^{1} \in \mathcal{M}\left(\Delta_{a_{1}}(r) \times \Omega_{2}\right)$ and $f_{a}^{2} \in \mathcal{M}\left(\Omega_{1} \times \Delta_{a_{2}}(r)\right)$ which coincide with $f$ on $(T \backslash S) \cap \Delta_{a}(r)$. Moreover, one sees that the function $f_{\mathcal{U}, \mathcal{V}}$ given by

$$
f_{\mathcal{U}, \mathcal{V}}:=f_{a}^{1} \quad \text { on } \Delta_{a_{1}}(r) \times \Omega_{2}, \quad f_{\mathcal{U}, \mathcal{V}}:=f_{a}^{2} \quad \text { on } \Omega_{1} \times \Delta_{a_{2}}(r),
$$

is well defined, meromorphic on the cross $X_{\mathcal{U}, \mathcal{V}}=\mathbb{X}\left(\Delta_{a_{1}}(r), \Delta_{a_{2}}(r) ; \Omega_{1}, \Omega_{2}\right)$, and $f_{\mathcal{U}, \mathcal{V}}=f$ on $(T \backslash S) \cap X_{\mathcal{U}, \mathcal{V}}$.

Using Remark 4.2, one can also prove the following. If $\mathcal{U}^{\prime} \subset D_{1}, \mathcal{V}^{\prime} \subset$ $D_{2}$ are arbitrary open sets and $f_{\mathcal{U}^{\prime}, \mathcal{V}^{\prime}}$ is the corresponding meromorphic function defined on the corresponding cross $X_{\mathcal{U}^{\prime}, \mathcal{V}^{\prime}}$, then $f_{\mathcal{U}, \mathcal{V}}=f_{\mathcal{U}^{\prime}, \mathcal{V}^{\prime}}$ on $X_{\mathcal{U}, \mathcal{V}} \cap X_{\mathcal{U}^{\prime}, \mathcal{V}^{\prime}}$. 
Let $A_{j}:=\bigcup_{\mathcal{U} \subset \Omega_{1}, \mathcal{V} \subset \Omega_{2}} \Delta_{a_{j}}(r)$ for $j=1,2$. It is clear that $A_{j}$ is an open dense set in $\Omega_{j}$. Then gluing all $f_{\mathcal{U}, \mathcal{V}}$ together, we obtain a function $\widehat{f}$ meromorphic on $X:=\mathbb{X}\left(A_{1}, A_{2} ; \Omega_{1}, \Omega_{2}\right)$ satisfying $\widehat{f}=f$ on $(T \backslash S) \cap X$. Finally, one applies Theorem 1.3 of [4] to $\widehat{f}$, and the conclusion of (ii) follows.

Proof of (i). In what follows, $\Sigma_{M}$ will denote the group of permutations of $\{1, \ldots, M\}$. Moreover, for any $\sigma \in \Sigma_{M}$ and under the hypothesis and in the notation of Lemma 4.1, we define

$$
S^{\sigma}:=\left\{z^{\sigma}: z \in S\right\} \quad \text { and } \quad \Omega^{\sigma}:=\Omega_{\sigma(1)} \times \ldots \times \Omega_{\sigma(M)},
$$

where

$$
z^{\sigma}:=\left(z_{\sigma(1)}, \ldots, z_{\sigma(M)}\right), \quad z \in \Omega=\Omega_{1} \times \ldots \times \Omega_{M} .
$$

If in the statement of Lemma 4.1, one replaces $S$ by $S^{\sigma}$ and $\Omega$ by $\Omega^{\sigma}$, then one obtains $\mathcal{S}^{\sigma}, S^{\sigma}\left(a_{\sigma(N)}\right), \ldots, S^{\sigma}\left(a_{\sigma(3)}, \ldots, a_{\sigma(N)}\right)$. The proof will be divided into three steps.

STEP 1: $N=2$. By (ii), for each pair of relatively compact pseudoconvex subdomains $\Omega_{j} \subset D_{j}(j=1,2)$ we obtain a polydisc $\Delta_{\Omega_{1}, \Omega_{2}} \subset \Omega_{1} \times \Omega_{2}$ and a function $f_{\Omega_{1}, \Omega_{2}} \in \mathcal{O}\left(\Delta_{\Omega_{1}, \Omega_{2}}\right)$ such that $f=f_{\Omega_{1}, \Omega_{2}}$ on $\left(\Delta_{\Omega_{1}, \Omega_{2}} \cap T\right) \backslash S$. A routine identity argument shows that any two functions $f_{\Omega_{1}, \Omega_{2}}$ coincide on the intersection of their domains of definition. Gluing $f_{\Omega_{1}, \Omega_{2}}$, we obtain the desired function $\widehat{f} \in \mathcal{O}\left(\bigcup \Delta_{\Omega_{1}, \Omega_{2}}\right)$.

SteP 2: $N=3$. Consider the following elements of $\Sigma_{3}$ :

$$
\begin{aligned}
\sigma_{1}:=\left(\begin{array}{lll}
1 & 2 & 3 \\
2 & 1 & 3
\end{array}\right), & \sigma_{2}:=\left(\begin{array}{lll}
1 & 2 & 3 \\
1 & 2 & 3
\end{array}\right), \\
\sigma_{3}:=\left(\begin{array}{lll}
1 & 2 & 3 \\
3 & 2 & 1
\end{array}\right), & \sigma_{4}:=\left(\begin{array}{lll}
1 & 2 & 3 \\
3 & 1 & 2
\end{array}\right) .
\end{aligned}
$$

Fix any subdomain $\Omega_{1} \times \Omega_{2} \times \Omega_{3} \subset D$ and pick any $a_{3} \in \mathcal{S}^{\sigma_{1}} \cap \mathcal{S}^{\sigma_{2}}$. Then by the definition, $\Omega_{1} \backslash S^{\sigma_{1}}\left(a_{3}\right)$ (resp. $\Omega_{2} \backslash S^{\sigma_{2}}\left(a_{3}\right)$ ) is of Baire category I in $\Omega_{1}$ (resp. $\Omega_{2}$ ).

Also, for any $a_{1} \in S^{\sigma_{1}}\left(a_{3}\right) \cap \mathcal{S}^{\sigma_{3}}$, we have int $S\left(a_{1}, \cdot, a_{3}\right)=\emptyset$ and the set $\Omega_{2} \backslash\left\{a_{2} \in \Omega_{2}: \operatorname{int} S\left(a_{1}, a_{2}, \cdot\right)=\emptyset\right\}$ is of Baire category I.

Similarly, for any $a_{2} \in S^{\sigma_{2}}\left(a_{3}\right) \cap \mathcal{S}^{\sigma_{4}}$, we have int $S\left(\cdot, a_{2}, a_{3}\right)=\emptyset$ and the set $\Omega_{1} \backslash\left\{a_{1} \in \Omega_{1}\right.$ : int $\left.S\left(a_{1}, a_{2}, \cdot\right)=\emptyset\right\}$ is of Baire category I.

Thus $f$ is well defined on the union $X$ of the following two subsets of $\Omega_{1} \times \Omega_{2} \times\left\{a_{3}\right\}:$

$$
\begin{aligned}
& \left\{\left(z_{1}, z_{2}, a_{3}\right): z_{1} \in S^{\sigma_{1}}\left(a_{3}\right) \cap \mathcal{S}^{\sigma_{3}}, z_{2} \in S^{\sigma_{3}}\left(z_{1}\right) \cap S^{\sigma_{2}}\left(a_{3}\right)\right\}, \\
& \left\{\left(z_{1}, z_{2}, a_{3}\right): z_{2} \in S^{\sigma_{2}}\left(a_{3}\right) \cap \mathcal{S}^{\sigma_{4}}, z_{1} \in S^{\sigma_{4}}\left(z_{2}\right) \cap S^{\sigma_{1}}\left(a_{3}\right)\right\} .
\end{aligned}
$$


Observe that by the definition in Lemma 4.1, $\Omega_{1} \backslash\left(S^{\sigma_{4}}\left(z_{2}\right) \cap S^{\sigma_{1}}\left(a_{3}\right)\right)$ (resp. $\left.\Omega_{2} \backslash\left(S^{\sigma_{3}}\left(z_{1}\right) \cap S^{\sigma_{2}}\left(a_{3}\right)\right)\right)$ is of Baire category I in $\Omega_{1}$ (resp. $\Omega_{2}$ ). By virtue of (5.2)-(5.3), the same conclusion also holds for the fibers $X\left(z_{1}, \cdot, a_{3}\right)$ and $X\left(\cdot, z_{2}, a_{3}\right), z_{1} \in S^{\sigma_{1}}\left(a_{3}\right) \cap \mathcal{S}^{\sigma_{3}}$ (resp. $\left.z_{2} \in S^{\sigma_{2}}\left(a_{3}\right) \cap \mathcal{S}^{\sigma_{4}}\right)$.

Let $\mathcal{U}_{j} \subset \Omega_{j}(j=1,2)$ be an arbitrary open subset. If $\Delta:=\Delta_{q}(r)$ is a polydisc, then we denote by $\lambda \Delta$ the polydisc $\Delta_{q}(\lambda r)$ for all $\lambda>0$. Repeating the Baire category argument already used in the proof of part (ii), one can show that there are a positive number $K$, polydiscs $\Delta_{j} \subset \mathcal{U}_{j}$, and subsets $Q_{\mathcal{U}_{1}, \mathcal{U}_{2}}^{1}$ of $S^{\sigma_{1}}\left(a_{3}\right) \cap \mathcal{S}^{\sigma_{3}}$ (resp. $Q_{\mathcal{U}_{1}, \mathcal{U}_{2}}^{2}$ of $\left.S^{\sigma_{2}}\left(a_{3}\right) \cap \mathcal{S}^{\sigma_{4}}\right)$ such that $\overline{Q_{\mathcal{U}_{1}, \mathcal{U}_{2}}^{j}}=\Delta_{j}, Q_{\mathcal{U}_{1}, \mathcal{U}_{2}}^{j}$ is of Baire category II, and $\sup _{2 \Delta_{2}}\left|f\left(\widetilde{a_{1}, \cdot, a_{3}}\right)\right| \leq K$ (resp. $\left.\sup _{2 \Delta_{1}}\left|f\left(\widetilde{\cdot, a_{2}, a_{3}}\right)\right| \leq K\right)$ for $a_{j} \in Q_{\mathcal{U}_{1}, \mathcal{U}_{2}}^{j}$.

Therefore, by applying Lemma 4.2 , we obtain a function $f_{a_{3}}=f_{\mathcal{U}_{1}, \mathcal{U}_{2}, a_{3}} \in$ $\mathcal{O}\left(\Delta_{1} \times \Delta_{2}\right)$ which extends $f\left(\cdot, \cdot, a_{3}\right)$ to $\Delta_{1} \times \Delta_{2} \times\left\{a_{3}\right\}$ for all $a_{3} \in \mathcal{S}^{\sigma_{1}} \cap \mathcal{S}^{\sigma_{2}}$.

Now let $\mathcal{U}_{j} \subset \Omega_{j}(j=1,2,3)$ be an arbitrary open subset. Since the set $\Omega_{3} \backslash\left(\mathcal{S}^{\sigma_{1}} \cap \mathcal{S}^{\sigma_{2}}\right)$ is of Baire category I, by using the previous discussion we can employ the Baire category argument already used in the proof of (ii). Consequently, there are a positive number $K$, polydiscs $\Delta_{j} \subset \mathcal{U}_{j}$, and subsets $Q_{\mathcal{U}_{1}, \mathcal{U}_{2}, \mathcal{U}_{3}}^{3}$ of $\mathcal{S}^{\sigma_{1}} \cap \mathcal{S}^{\sigma_{2}}$ such that $\overline{Q_{\mathcal{U}_{1}, \mathcal{U}_{2}, \mathcal{U}_{3}}^{3}}=\Delta_{3}, Q_{\mathcal{U}_{1}, \mathcal{U}_{2}, \mathcal{U}_{3}}^{3}$ is of Baire category II, and $\sup _{2 \Delta_{1} \times 2 \Delta_{2}}\left|f_{a_{3}}(\cdot, \cdot)\right| \leq K$ for $a_{3} \in Q_{\mathcal{U}_{1}, \mathcal{U}_{2}, \mathcal{U}_{3}}^{3}$.

By changing the role of $1,2,3$ and by taking smaller polydiscs, we obtain in the same way subsets $Q_{\mathcal{U}_{1}, \mathcal{U}_{2}, \mathcal{U}_{3}}^{j} \subset \Delta_{j}(j=1,2)$ with similar properties.

For $j \in\{1,2,3\}$ consider the following subsets of $T$ :

$$
\begin{aligned}
T_{j}:=\left\{a=\left(a_{1}, a_{2}, a_{3}\right):\right. & \\
& a_{k} \in \Delta_{k}, a_{l} \in \Delta_{l}, a_{j} \in Q_{\mathcal{U}_{1}, \mathcal{U}_{2}, \mathcal{U}_{3}}^{j},\{k, l, j\}=\{1,2,3\} \text { and } \\
& \operatorname{either}_{\left.\operatorname{int}_{\mathbb{C}^{n}} S\left(\cdot, a_{k}, a_{j}\right)=\emptyset \operatorname{or}_{\mathbb{n}^{n_{k}}} S\left(a_{l}, \cdot, a_{j}\right)=\emptyset\right\} .}
\end{aligned}
$$

One next proves that

$$
f(a)=f_{a_{j}}\left(a_{k}, a_{l}\right), \quad a \in T_{1} \cup T_{2} \cup T_{3} .
$$

Indeed, let $a=\left(a_{1}, a_{2}, a_{3}\right) \in T_{3}$ with $\operatorname{int} S\left(\cdot, a_{2}, a_{3}\right)=\emptyset$. In view of (5.2), we can choose a sequence $\left(z_{1}^{n}\right)_{n=1}^{\infty} \rightarrow a_{1}$ and for every $n \geq 1$ a sequence $\left(z_{2}^{n, m}\right)_{m=1}^{\infty} \rightarrow a_{2}$ such that $\left(z_{1}^{n}, z_{2}^{n, m}, a_{3}\right) \in X$ for all $n, m \in \mathbb{N}$. Clearly, $f\left(z_{1}^{n}, z_{2}^{n, m}, a_{3}\right)=f_{a_{3}}\left(z_{1}^{n}, z_{2}^{n, m}\right)$. Therefore,

$$
f(a)=\lim _{n \rightarrow \infty} f\left(z_{1}^{n}, a_{2}, a_{3}\right)=\lim _{n \rightarrow \infty} \lim _{m \rightarrow \infty} f\left(z_{1}^{n}, z_{2}^{n, m}, a_{3}\right)=f_{a_{3}}\left(a_{1}, a_{2}\right) .
$$

Now, we wish to glue the three functions $f_{a_{j}}(j=1,2,3)$ together. Since the family $\left\{f_{a_{j}}: a_{j} \in Q_{\mathcal{U}_{1}, \mathcal{U}_{2}, \mathcal{U}_{3}}^{j}\right\}$ is normal, we define an extension $f_{j}$ of $f_{a_{j}}(j=1,2,3)$ to $\Delta:=\Delta_{1} \times \Delta_{2} \times \Delta_{3}$ as follows.

Let $\{j, k, l\} \in\{1,2,3\}$ and for $z=\left(z_{1}, z_{2}, z_{3}\right) \in \Delta$, choose a sequence $\left(a_{j}^{n}\right)_{n=1}^{\infty} \subset Q_{\mathcal{U}_{1}, \mathcal{U}_{2}, \mathcal{U}_{3}}^{j}$ such that $\lim _{n \rightarrow \infty} a_{j}^{n}=z_{j}$ and the sequence $\left(f_{a_{j}}\right)_{n=1}^{\infty}$ 
converges uniformly on compact subsets of $\Delta_{k} \times \Delta_{l}$. We let

$$
f_{j}(z):=\lim _{n \rightarrow \infty} f_{a_{j}^{n}}\left(a_{k}^{n}, a_{l}^{n}\right),
$$

for any sequence $\left(\left(a_{k}^{n}, a_{l}^{n}, a_{j}^{n}\right)\right)_{n=1}^{\infty} \subset T_{j} \rightarrow z$ as $n \rightarrow \infty$.

Let us first check that the functions $f_{j}$ are well defined. Indeed, this will follow from the estimate

$$
\begin{aligned}
& \left|f_{a_{j}}\left(a_{k}, a_{l}\right)-f_{b_{j}}\left(b_{k}, b_{l}\right)\right| \leq C K|a-b|, \\
& \quad a=\left(a_{k}, a_{l}, a_{j}\right), b=\left(b_{k}, b_{l}, b_{j}\right) \in T_{j} .
\end{aligned}
$$

Here $C$ is a constant that depends only on $\Delta$. It now remains to prove (5.6) for example in the case $j=3$. To do this, let $z=\left(z_{1}, z_{2}, z_{3}\right), w=$ $\left(w_{1}, w_{2}, w_{3}\right) \in T_{3}$. Then by $(5.2)$ and (5.3), one can choose $a_{1}, a_{1}^{\prime} \in \Delta_{1}$ and $a_{2} \in \Delta_{2}$ such that

$$
\begin{aligned}
& \left(a_{1}, a_{2}, z_{3}\right),\left(a_{1}^{\prime}, a_{2}, z_{3}\right) \in T_{2} \cap T_{3}, \\
& \left|z-\left(a_{1}, a_{2}, z_{3}\right)\right| \leq 2|z-w|, \quad\left|w-\left(a_{1}^{\prime}, a_{2}, z_{3}\right)\right| \leq 2|z-w| .
\end{aligned}
$$

Write

$$
\begin{aligned}
\left|f_{3}(z)-f_{3}(w)\right| \leq & \left|f_{3}(z)-f_{3}\left(a_{1}, a_{2}, z_{3}\right)\right| \\
& +\left|f_{3}\left(a_{1}^{\prime}, a_{2}, z_{3}\right)-f_{3}(w)\right|+\left|f_{2}\left(a_{1}, a_{2}, z_{3}\right)-f_{2}\left(a_{1}^{\prime}, a_{2}, z_{3}\right)\right| .
\end{aligned}
$$

Since $\sup _{2 \Delta_{1} \times 2 \Delta_{2}}\left|f_{z_{3}}\right| \leq K, \sup _{2 \Delta_{1} \times 2 \Delta_{2}}\left|f_{w_{3}}\right| \leq K$ and $\sup _{2 \Delta_{1} \times 2 \Delta_{3}}\left|f_{a_{2}}\right|$ $\leq K$, applying Schwarz's lemma to each term on the right side of the latter estimate and using (5.7) we obtain the desired estimate (5.6).

From the construction (5.5) above, $f_{j}\left(\cdot, \cdot, z_{j}\right) \in \mathcal{O}\left(\Delta_{k} \times \Delta_{l}\right)$. Moreover, a routine identity argument using (5.2) and (5.3) shows that $f_{1}=f_{2}=f_{3}$. Finally, define

$$
\widehat{f}_{\mathcal{U}_{1}, \mathcal{U}_{2}, \mathcal{U}_{3}}(z)=f_{1}(z)=f_{2}(z)=f_{3}(z), \quad z \in \Delta ;
$$

then $\widehat{f}_{\mathcal{U}_{1}, \mathcal{U}_{2}, \mathcal{U}_{3}}$ extends $f$ holomorphically from $T_{1} \cup T_{2} \cup T_{3}$ to $\Delta$. Again an identity argument as in (5.4) shows that $\widehat{f}_{\mathcal{U}_{1}, \mathcal{U}_{2}, \mathcal{U}_{3}}=f$ on $(T \cap \Delta) \backslash S$. Gluing $\widehat{f}_{\mathcal{U}_{1}, \mathcal{U}_{2}, \mathcal{U}_{3}}$ for all $\mathcal{U}_{1}, \mathcal{U}_{2}, \mathcal{U}_{3}$ together we obtain the desired extension function $\widehat{f}$. Hence the proof is complete in this case.

StEP 3: $N \geq 4$. The general case uses induction on $N$. Since the proof is very similar to the case $N=3$ making use of Lemmas 4.1 and 4.3 and using the inductive hypothesis for $N-1$, we leave the details to the reader.

\section{References}

[1] M. Jarnicki and P. Pflug, Extension of Holomorphic Functions, de Gruyter Exp. Math. 34, Walter de Gruyter, 2000.

[2] - - - An extension theorem for separately holomorphic functions with analytic singularities, Ann. Polon. Math. 80 (2003), 143-161. 
[3] M. Jarnicki and P. Pflug, An extension theorem for separately holomorphic functions with pluripolar singularities, Trans. Amer. Math. Soc. 355 (2003), 1251-1267.

[4] - - - An extension theorem for separately meromorphic functions with pluripolar singularities, Kyushu J. Math. (2003), to appear; arXiv:math.CV/0209207 v2, $1-11$.

[5] M. Klimek, Pluripotential Theory, London Math. Soc. Monogr. 6, Oxford Univ. Press, 1991.

[6] Nguyên Thanh Vân et J. Siciak, Fonctions plurisousharmoniques extrémales et systèmes doublement orthogonaux de fonctions analytiques, Bull. Sci. Math. 115 (1991), 235-244.

[7] Nguyên Thanh Vân et A. Zériahi, Une extension du théorème de Hartogs sur les fonctions séparément analytiques, in: Analyse Complexe Multivariable : Récents Développements (Guadeloupe, 1988), A. Meril (ed.), EditEl, Rende, 1991, 183-194.

[8] W. Rothstein, Ein neuer Beweis des Hartogsschen Hauptsatzes und seine Ausdehnung auf meromorphe Funktionen, Math. Z. 53 (1950), 84-95.

[9] E. Sakai, A note on meromorphic functions in several complex variables, Mem. Fac. Sci. Kyusyu Univ. 11 (1957), 75-80.

[10] Y. T. Siu, Techniques of Extension of Analytic Objects, Lecture Notes in Pure and Appl. Math. 8, Marcel Dekker, 1974.

Institut für Mathematik

Carl von Ossietzky Universität Oldenburg

Postfach 2503, D-26111, Oldenburg, Germany

E-mail: pflug@mathematik.uni-oldenburg.de nguyen@mathematik.uni-oldenburg.de 